\title{
Obstructive Jaundice - The Radiological Study in Assessing the Level and Cause
}

\author{
Antony Jean ${ }^{1}$, Murali Krishna ${ }^{2}$, Prakash Manikka Lakshmanan ${ }^{3}$, Annitha Elavarasi Jayamohan ${ }^{4}$ \\ ${ }^{1}$ Assistant Professor, Department of Radio-diagnosis, Dhanalakshmi Srinivasan Medical College \& Hospital, Perambalur, ${ }^{2}$ Consultant Radiologist, Department of \\ Radio-diagnosis, Apollo Hospital, Chennai, ${ }^{3}$ (Retired)Professor \& HOD, Department of Radio-diagnosis, MGMCRI, Pondicherry, ${ }^{4}$ Consultant Radiologist, \\ Department of Radio-diagnosis, Mallaya Hospital, Bangalore.
}

\section{Abstract}

Background: Obstructive jaundice is a common clinical problem. The main objective of the study is to determine the accuracy of MRCP in the evaluation of patients with obstructive jaundice. Subjects and Methods: The study was conducted in the department of Radio Diagnosis, MGMC\&RI, for a period of 2 years. Thirty three patients were included in the study. All the patients were referred to the department of radio diagnosis with the clinical suspicion of obstructive jaundice and elevated serum bilirubin levels. MRCP was done in all the patients. Three experienced radiologists reviewed the images separately and evaluated the cause and site of obstruction in these patients. The accuracy of each modality was analyzed statistically and correlation was made with the surgical findings or histopathological reports. Results: Of the thirty three patients, seventeen patients had benign causes of obstructive jaundice while sixteen patients had malignant causes of obstructive jaundice. MRCP had an accuracy of $97 \%$ in detecting the cause of obstructive jaundice. Conclusion: With the introduction of MRI guided interventions it may soon be possible in the near future to use MRCP for diagnostic and therapeutic applications in biliary tract and pancreatic pathology.

Keywords: Obstructive jaundice, Radiological, MRCP.

Corresponding Author: Dr. Murali Krishna, Consultant Radiologist, Department of Radio-diagnosis, Apollo Hospital, Chennai.

Received: May 2019

Accepted: June 2019

\section{Introduction}

Obstructive jaundice is a common clinical problem. It has been documented as one of the leading cause of increased mortality and morbidity. Though clinical data such as history, physical examination, and laboratory tests can differentiate between intra-hepatic \& extra-hepatic obstruction in $90 \%$ of patients, the cause and site of obstruction is diagnosed by imaging modalities. The main goals of any imaging procedure in obstructive jaundice are to confirm the presence of obstruction, its location, extent, probable cause, and it should also attempt to obtain a map of the biliary tree that will help the surgeon or the interventionist to determine the best approach to each individual case. ${ }^{[1,2]}$

The commonly used imaging modalities include Ultrasonography (USG), Computed Tomography (CT), Endoscopic Retrograde Cholangiopancreatography (ERCP) and Magnetic Resonance Cholangiopancreatography (MRCP). Percutaneous Transhepatic Cholangiography (PTC) is used for drainage procedures. ${ }^{[3,4]}$

Normal intra hepatic bile ducts measure less than $3 \mathrm{~mm}$ in diameter, fewer in number and are randomly scattered throughout the liver 2. They are linear water density structures seen along one side of portal vein. Towards the hilum they unite to form right and left hepatic ducts which have a constant location just anterior to main portal vein bifurcation 3. Normally peripheral intra hepatic bile ducts are not visualized on MR imaging. ${ }^{[1,5,6]}$

Two right $\&$ left hepatic unite near the right end of the porta hepatis to form the common hepatic duct; this is usually imaged as a round or elliptical structure sitting anterior and often slightly lateral to main portal vein. The common hepatic duct lies to the right and lateral to the proper hepatic artery, and usually measures 3 to $6 \mathrm{~mm}$ in short axis diameter. The wall of CHD is normally visualized and measures less than $1.5 \mathrm{~mm}^{[7,8]}$

The Gall bladder is a pear shaped sac partly contained in a fossa on the inferior surface of the right hepatic lobe. It is 7 to $10 \mathrm{~cm}$ long, $3 \mathrm{~cm}$ broad at its widest part and 30 to $50 \mathrm{ml}$ in capacity. It is divided into a fundus, body and neck. ${ }^{[9]}$ Obstructive jaundice is the commonest presentation in patients with biliary obstruction. The role of imaging is crucial for detection of site and cause of obstruction. In case of malignant obstruction, characterization of the lesion and staging of the tumor is crucial to decide optimal management of the disease. These patients in general are subjected to diagnostic US followed by CECT. It has been proposed that when complete MR imaging is performed including $\mathrm{T} 1$ and $\mathrm{T} 2$ weighted images and Gadolinium enhanced MR along with MRCP, it has the capacity to provide all in one evaluation of the suspected obstructive lesions, obviating the need for any other investigation such 
as $\mathrm{CT} / \mathrm{PTC} / \mathrm{ERCP} .^{[10-15]}$

MRCP uses heavily T2Weighted sequences to take advantage of the inherent contrast effect of bile. Overlap between high signals from the pancreaticobiliary system and from the gastrointestinal tract (GIT) (stomach, duodenum and proximal intestine), is a recognized limitation of MRCP and may mimic pathology. Several studies have shown that the administration of a negative oral contrast material, before performing a MRCP will improve image quality and provide good visualization of the bile and pancreatic ducts without superimposed high signal from the GIT. ${ }^{[16]}$

A number of negative oral contrast agents are available for MR imaging of the abdomen and pelvis. Examples include Gadopentate dimeglumine, ferric ammonium citate53, manganese chloride, Kaolinate, antacid, barium sulphate and ferric particles. Many of these are relatively unpalatable, become too diluted in the GIT or are expensive. This study demonstrates that Pineapple Juice may be used as a negative oral contrast agent to improve the quality of MRCP images, using a simple technique. It is of particular benefit in improving the conspicuity of the pancreatic duct, and also improving the visualization of the common bile duct, ampulla, intrahepatic and common hepatic ducts on MRCP images. ${ }^{[14]}$

Several studies done on different modalities have claimed superiority of different modalities. ${ }^{[5,9]}$ This study aims to compare the commonly available modalities in the Indian set up and prove the efficacy of the individual modalities. The study also hopes to formulate a correct investigative protocol for patients with obstructive jaundice.

The present study is done to evaluate the diagnostic accuracy of Magnetic Resonance Cholangiopancreatography (MRCP) in assessing the level and cause of obstruction in patients with Obstructive Jaundice.

\section{Subjects and Methods}

This study was conducted in the Department of Radio
Diagnosis, MGMC\&RI, Pondicherry. A total no of thirty three patients suffering from obstructive jaundice of all age groups and either sex were included in this study.

\section{Inclusion criteria}

a) Patient clinically diagnosed as suffering from obstructive jaundice.

b) Total Bilirubin greater than $5 \mathrm{mg} / \mathrm{dl}$.

c) Patient referred to the Department of Radio Diagnosis for further investigation.

\section{Exclusion criteria}

a) Claustrophobia

b) Renal insufficiency

c) Pregnancy

d) MR incompatibility

The study protocol was approved by the ethical committee at Sri Balaji Vidyapeeth University and all the patients gave informed consent to participate.

\section{Patient preparation:}

- All the patients were instructed to fast over night prior to examination.

- Renal functional status of all the patients was noted before undergoing contrast CT.

- Clinical history of all the patients was elicited to rule out previous contrast reactions/allergies.

- All the metallic belongings removed prior to the examination.

\section{Procedure:}

All the patients in the study underwent USG examination first followed by MRCP and finally CT. This order is followed so that there is no interference of image quality from the previous study. MRCP was performed on Philips Healthcare Intera 1.5 Tesla MRI Scanner. All images were obtained with breath holding and parameters were individualized. Detailed parameters of each sequence are summarized below.

\begin{tabular}{|c|c|c|c|c|c|c|c|}
\hline Sequence & TR (ms) & TE (ms) & No of Slices (mm) & Slice Thickness (mm) & Gap (mm) & Matrix & FOV (mm) \\
\hline MRCP 3D HR & 1204 & 650 & 110 & 1.0 & 0.8 & 512 & 266 \\
\hline Ssh MRCP RAD & 8000 & 800 & 12 & 40 & 0.4 & 512 & 300 \\
\hline Ssh SPAIR COR & 465 & 80 & 25 & 5 & 0.5 & 486 & 330 \\
\hline Ssh SPAIR TRA & 425 & 80 & $40 / 35$ & 5 & 0.5 & 420 & 330 \\
\hline Ssh SPAIR SAG & 462 & 80 & 40 & 5 & 0.5 & 384 & 270 \\
\hline T2TSE HR TRA & 2504 & 100 & 36 & 5 & 0.5 & 512 & 360 \\
\hline T1W 3D TSE & 10 & 4.6 & 80 & 1.0 & 0.1 & 256 & 375 \\
\hline
\end{tabular}

\section{The following Parameters were studied for MRCP}

1. Level of obstruction(four Anatomical Segments)

- Hepatic

- Suprapancreatic

- Pancreatic

- Ampullary

2. Presence of bile duct calculi

- Non visualized

- Definitely visualized.

3. Status of CBD

- Smooth tapering
- Abrupt end

- Rounded

- Irregular

4. Degree of dilatation of intra hepatic biliary radicles.

5. Gall bladder pathology including size, wall, stones.

6. Dilatation of pancreatic duct.

7. Pancreatic atrophy, calcifications, and pseudocysts.

8. Invasion of viscera, fascial planes.

9. Presence of metastasis.

Then classification of imaging findings as benign or malignant cause of obstructive jaundice is based on 
following scale of confidence.

\section{Definitely benign:}

Biliary duct dilatation with a visible stone in the duct with no associated mass or stricture.

\section{Probably benign:}

Cystic dilatation of bile duct. Pancreatico-biliary duct dilatation considered benign (i.e. Sign of chronic pancreatitis).

\section{Inconclusive:}

Not confidently diagnosed as benign or malignant.

\section{Probablv malignant:}

Iso-Hypo enhancing mass(for CT only) with indirect signs of tumor such as duct dilatation with ductal cut-off adjacent to the mass or atrophic distal parenchyma or pancreato biliary dilatation considered malignant without sign of a mass or lesion in pancreatic head without duct dilatation.

\section{Definitely malignant:}

Mass in the pancreatic head with consistent duct dilatation. Isolated CBD dilatation with an abrupt narrowing located cranial to the level of mass lesion.

\section{Results}

The age group of the patients varied from 21 to 86 . The average age of the patients in the study was 52 . There was no obvious sex predilection in the patients affected with obstructive jaundice. Among the sixteen patients with malignant lesions, MRCP had diagnosed all sixteen of them. In diagnosis the site of obstruction, MRCP was accurate in all thirty three patients. The statistics were analyzed using SPS Statistical Software. The modalities were compared using CHI-SQUARE tests.

Table 1: Age distribution of various pathologies in studied Population.

\begin{tabular}{|l|l|l|}
\hline Age Group & No Of Cases & Percentage (\%) \\
\hline Children(0-12yrs) & 0 & 0 \\
\hline $\begin{array}{l}\text { Adolescent\& young } \\
\text { adults(13-30yrs) }\end{array}$ & 5 & 15 \\
\hline Adults(31-60yrs) & 15 & 45 \\
\hline $\begin{array}{l}\text { Geriatric } \\
\text { patient( }>60 \mathrm{yrs})\end{array}$ & 13 & 40 \\
\hline Total & 33 & 100 \\
\hline
\end{tabular}

The [Table 1] shows that most of the patients affected with obstructive jaundice are over the age of 30 yrs. The mean age of the patients was 52 . More than $60 \%$ of the patients affected were over the age of 50 yrs. The average age of patients with benign lesions was $45 \mathrm{yrs}$ while that of malignant lesions was $59.8 \mathrm{yrs}$.

\section{Table 2: Sex incidence in the studied population.}

\begin{tabular}{|l|l|l|}
\hline Sex & No Of Cases & Percentage (\%) \\
\hline Male & 17 & 52 \\
\hline Female & 16 & 48 \\
\hline Total & 33 & 100 \\
\hline
\end{tabular}

Table 3: Benign versus Malignant causes of obstructive jaundice in the studied population.

\begin{tabular}{|l|l|l|}
\hline Type of lesion & No of cases & Percentage (\%) \\
\hline Benign & 17 & 52 \\
\hline Malignant & 16 & 48 \\
\hline Total & 33 & 100 \\
\hline
\end{tabular}

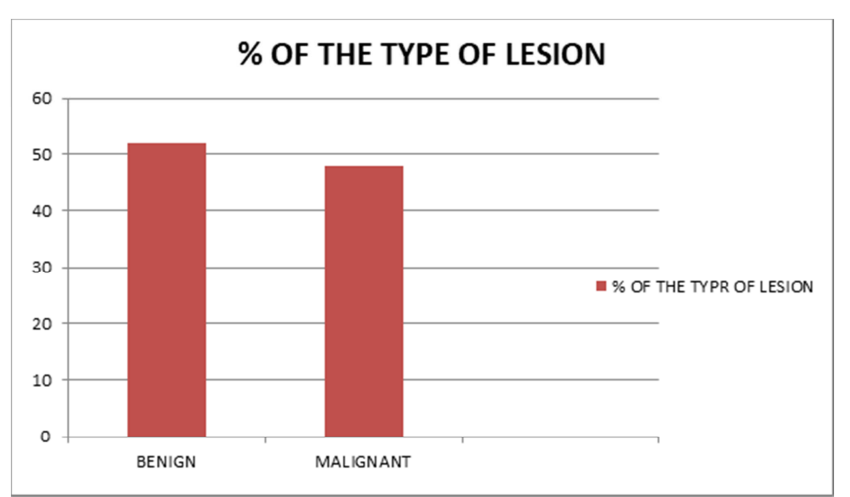

We observe from the above [Table 3] that there is almost equal predilection for both benign and malignant causes of obstructive jaundice.

Table 4: Various causes of obstructive jaundice studied in the population.

\begin{tabular}{|l|l|l|}
\hline Pathology & No Of Cases & Percentage (\%) \\
\hline Anatomic variants & 1 & 3 \\
\hline GB and CBD calculi & 8 & 25 \\
\hline Benign Stricture & 5 & 15 \\
\hline Cholangitis & 1 & 3 \\
\hline Ca Head of Pancreas & 4 & 12 \\
\hline Periampullary Ca & 3 & 9 \\
\hline Cholangiocarcinoma & 3 & 9 \\
\hline Ca GB & 3 & 9 \\
\hline Klatskins tumor & 2 & 6 \\
\hline Benign compression & 2 & 6 \\
\hline Metastatic compression & 1 & 3 \\
\hline Total & 33 & 100 \\
\hline
\end{tabular}

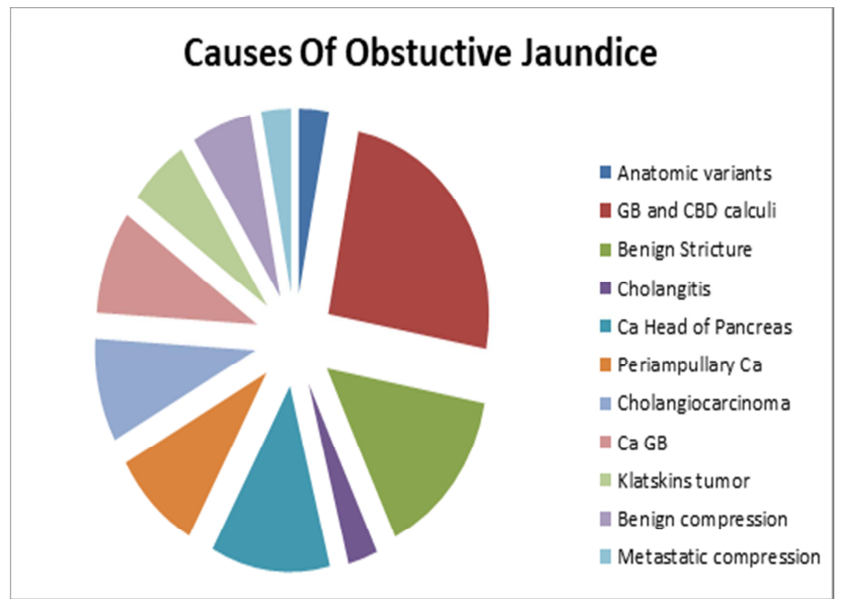

The illustration gives us a graphic representation of the common causes of obstructive jaundice. CBD calculi are the most common cause of obstructive jaundice followed by benign strictures and $\mathrm{Ca}$ Head of Pancreas. 
Table 5: Benign causes of obstructive jaundice in the studied population.

\begin{tabular}{|l|l|l|}
\hline Pathology & No Of Cases & Percentage (\%) \\
\hline Anatomic variants & 1 & 6 \\
\hline GB and CBD calculi & 8 & 47 \\
\hline Benign Stricture & 5 & 29 \\
\hline Cholangitis & 1 & 6 \\
\hline Benign compression & 2 & 12 \\
\hline Total & 17 & 100 \\
\hline
\end{tabular}

Benign Causes - \%

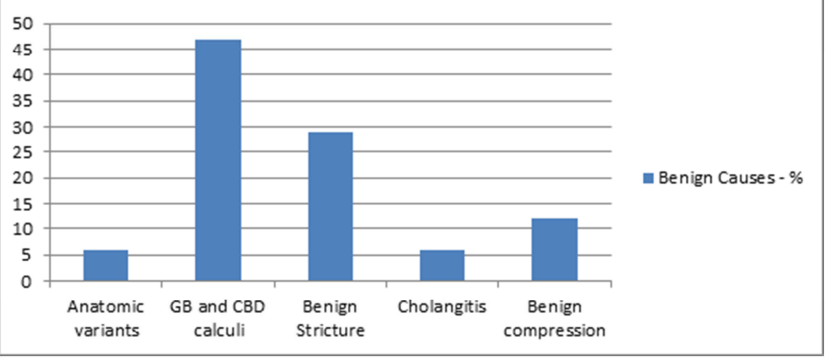

From the above [Table 5] and illustration it is clearly seen that GB and CBD calculi are the most common benign cause of obstructive jaundice.

Table 6: Malignant causes of obstructive jaundice in the studied population.

\begin{tabular}{|l|l|l|}
\hline Pathology & No Of Cases & Percentage (\%) \\
\hline Ca Head of Pancreas & 4 & 25 \\
\hline Periampullary Ca & 3 & 19 \\
\hline Cholangiocarcinoma & 3 & 19 \\
\hline Ca GB & 3 & 19 \\
\hline Klatskins tumor & 2 & 12 \\
\hline Metastatic compression & 1 & 6 \\
\hline Total & 16 & 100 \\
\hline
\end{tabular}

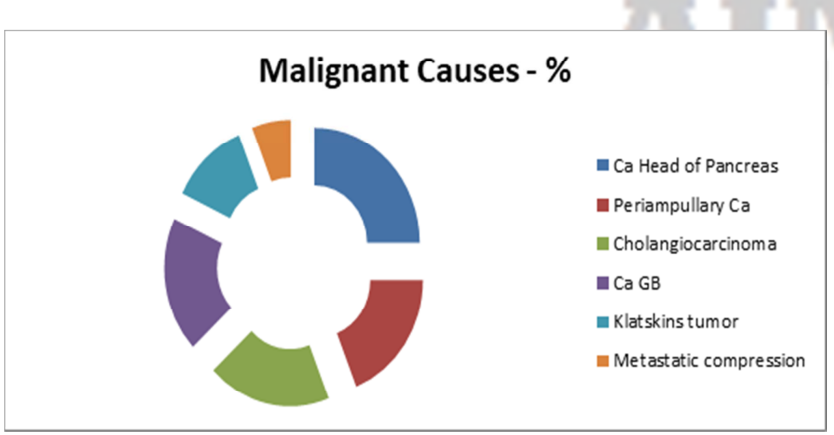

From the above [Table 6] and illustration we can see that carcinoma of the head of pancreas is the commonest malignant cause of obstructive jaundice.

\section{Discussion}

Diagnosing patients with suspected biliary or pancreatic pathologies in their early stage is almost importance in patient care and management. Knowledge of the advantages and disadvantages of each technique are needed to determine the appropriate work up of patients with these pathologies.

With the introduction of MR Cholangiopancreatography for the diagnosis of biliary and pancreatic ductal pathologies, invasive procedure like ERCP can be avoided solely for the purpose of diagnosis. ${ }^{[17]}$

In our study thirty three patients suffering from obstructive jaundice were studied. Most of the patients presented with jaundice and abdominal pain. Icterus was the most common sign followed by passing of white stools and itching. ${ }^{[18,19]}$

All the patients have a Total Bilirubin of more than $5 \mathrm{mg} / \mathrm{dl}$. It was noticed that in chronic obstructive cases and in patients with malignancy the Total Bilirubin was very elevated and was more than $10 \mathrm{mg} / \mathrm{dl}$. The alkaline phosphatase level was also elevated and was more than $100 \mathrm{U} / \mathrm{L}$.

The youngest patient in the study was twenty one years old female who was suffering from cholelithiasis with distal CBD stricture. The oldest patient was eighty six years old and was suffering from periampullary carcinoma. The average age of patients with benign lesions was in the fourth decade while that of malignant lesions was in the sixth decade.

Stricture disease was diagnosed in 4 patients. MRCP clearly showed benign nature of stricture in all four cases approaching $100 \%$ accuracy. MRCP showed clearly the length of the stricture segment very well and differentiated stricture as malignant and benign. Histopathology examination of the resected specimen revealed benign nature of obstruction. Our study is in concordance with Bhatt et al ${ }^{[20]}$ In their study they found $100 \%$ accuracy for $\mathrm{MRCP}$ in diagnosing benign CBD stricture. One case of cholangitis has been diagnosed wrongly as malignant stricture in MRCP, which histology proved to be a benign lesion.

One case of anatomical variant, a case of choledochal cyst was present in our study. Our study is in concordance with Bhatt et al, ${ }^{[20]}$ in their study they found $100 \%$ accuracy for $\mathrm{MRCP}$ in diagnosing anatomical variants.

Among the malignant lesions there were 4 cases of Head of Pancreas tumour and 3 cases of Periampullary Ca. MRCP accurately diagnosed all the seven cases. Though MRCP alone could not clinch the diagnosis a few sequences of MRI was required to diagnose accurately the malignant lesions. Our study is in concordance with Anderrson et $\mathrm{al},{ }^{[21]} 2005$; in their study they found $90 \%$ accuracy for MR and $80 \%$ accuracy for $\mathrm{CT}$ in diagnosing periampullary growth.

In 4 patients with extrahepatic Cholangiocarcinoma MRCP diagnosed all four cases with $100 \%$ accuracy with the help of conventional MRI, thus approaching $100 \%$ accuracy for MR with MRCP. When studying correlation between imaging findings and final diagnosis we found a stricture with malignant characteristics at MRCP to be the most predictive sign of malignancy. Our study is in accordance with Andersson et al ${ }^{[21]} 2005$; found that among MR with MRCP strictures with malignant characteristics at MRCP were the only independent predictor of malignancy.

Two patients were diagnosed to have klatskins tumour, and the accuracy of two modalities remain $100 \%$.Our study is in concordance with Bhatt et al 2005, ${ }^{[20]}$ in their study they found accuracy of $100 \%$ for MRCP alone in diagnosing klatskins tumour. Thus our study is in concordance with 
Soto et al, ${ }^{[22]}$ they inferred that Spiral CT less accurate than MR cholangiography in evaluation of Klatskins tumor in relation to extent of tumour as CT has less z axis resolution. Among 3 patients with Ca of Gallbladder 2 patients were diagnosed accurately by MRCP. The third case was associated with choledocholithiasis and was wrongly diagnosed as benign lesion.

ERCP is considered the standard of reference for imaging patients with obstructive jaundice, as it provides high resolution images of biliary tree and pancreatic duct. A great advantage of ERCP is its ability to perform therapeutic interventional procedures, including stone removal, stricture dilatation, and stent placement which will relieve obstruction. It requires a highly skilled and experienced endoscopist. Technical limitations can lead to unsuccessful examination. It may fail to show biliary tree proximal to severe obstruction. It is associated with significant post procedure morbidity and mortality. It cannot be performed in critically ill patient.

\section{Conclusion}

Of the thirty three patients, seventeen patients had benign causes of obstructive jaundice while sixteen patients had malignant causes of obstructive jaundice. MRCP had an accuracy of $97 \%$ in detecting the cause of obstructive jaundice. With the introduction of MRI guided interventions it may soon be possible in the near future to use MRCP for diagnostic and therapeutic applications in biliary tract and pancreatic pathology.

\section{References}

1. L.Van Hoe, D.Vanbeckevoort, K.Mermuys, W.Van Steenbergen, MR Cholangio-pancreatography, Atlas with Cross-Sectional Imaging Correlation, Second Edition, October 2005.

2. Koenraad J. Mortele and Pablo Ros: Anatomic variants of Biliary Tree: MR Cholangiographic findings and Clinical Applications. AJR 2001; 177: 389-394

3. Koichi Hirao et al: Evaluation of Aberrant Bile Ducts before Laparoscopic Cholecystectomy.AJR2000;175: 713-720.
4. Cotran, Kumar and Collins: Robbins Basic Pathology VIII edition, W B Saunders, Philadelphia, 2005.

5. Harrison's Principles of Internal Medicine, Volume 2, 17th edition, 2008.

6. Todani T, WAtanable T, Narusue $\mathrm{M}$ et al: Congenital bile duct cysts Classification, operative procedures and review of 37 cases, AM J. Surg. 1997.

7. Joseph K.T. Lee, Stuart S. Sagel et al: Computed Body Tomography with MRI correlation, 3rd edition, volume 2, Philadelphia, 1998.

8. Robert N. Gibson, Eugene Yeung, Jeremy N. Thompson, et al: Bile Duct Obstruction: Radiologic Evaluation of Level ,Cause, and Tumour resectability . Radiology1986; 160: 43-47.

9. Sirini Malini and John Sabel: Ultrasound in Obstructive Jaundice. Radiology 1981; 139: 635-645.

10. Carl M Bloom, Bernard Langer et al: Role of USG in the Detection, Characterization and Staging of Cholangiocarcinoma. Radiographics; 1984:1199-1218

11. Hyun Ju Lee, Ah Young Kim,et al: Three-dimensional Ultrasonography Using the Minimum Transparent Mode In Obstructive Biliary Diseases . J Ultrasound Med 21:443-453, 2002

12. Cesar S. Pedrosa, Rafael Casanova, et al: Computed Tomography in Obstructive Jaundice. Radiology 1981; 139: 635-645.

13. Threasa H. Reiman, Dennis Balfe, et al: Suprapancreatic Biliary Obstruction : CT Evaluation. Radiology 1987; 163: 49-56.

14. Grainger \& Allisons Diagnostic Radiology. A Textbook of Medical Imaging, Fifth Edition, Volume 1, Philadelphia : Elseiver, pg 763788.

15. Caroline Reinhold and Patrice M. Bret: Current status of MRCP. AJR1996; 166: 1285-1295

16. G.J. Robinson et al: MRCP in obstructive jaundice. Radiology 1997 , Imaging, Science \& Oncology, 124.

17. Matthew A. Barish et al: MRCP: Efficacy of 3-D Turbo spin- echo technique.AJR 1995; 165: 295-301.

18. Jorge A, Soto et al: MRCP: Findings on 3D fast spin-echo imaging. AJR1995; 165: 1397-1401.

19. Caroline Reinhold and Patrice M. Bret: Current status of MRCP. AJR1996; 166: 1285-1295.

20. Bhatt C, Shah P.S, Prajapati H.J, et al: Comparison of Diagnostic Accuracy between USG and MRCP in Biliary and Pancreatic Pathology. Ind J Radiol Imag 2005; 5:2:177-181.

21. Andersson M, Kostic S, Johansson M, Lundell L, Asztely M, et al: MRI combined with MR cholangiopancreatography versus helical CT in the evaluation of patients with suspected periampullary tumors: a prospective comparative study. Acta Radiologica 2005; 46: 16-27.

22. Soto et al: comparision of three dimensional fast spin echo and single and multisection half fourier rapid acquisition with relaxation enhancement sequences. Radiology 2000;215:737-745.

Copyright: (C) the author(s), publisher. Asian Journal of Medical Radiological Research is an Official Publication of "Society for Health Care \& Research Development". It is an open-access article distributed under the terms of the Creative Commons Attribution Non-Commercial License, which permits unrestricted non-commercial use, distribution, and reproduction in any medium, provided the original work is properly cited.

How to cite this article: Jean A, Krishna M, Lakshmanan PM, Jayamohan AE. Obstructive Jaundice - The Radiological Study in Assessing the Level and Cause. Asian J. Med. Radiol. Res. 2019;7(1):99-103.

DOI: dx.doi.org/10.21276/ajmrr.2019.7.1.22

Source of Support: Nil, Conflict of Interest: None declared 